تأثير الإضافات ( الرابطة ـ الملدنة ــ المزيته) على الخصائص الفيزياوية للكاؤولين

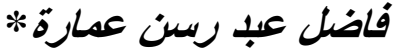

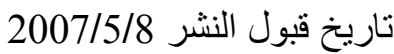

الخلاصة:

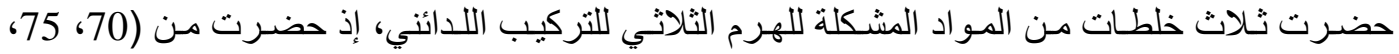



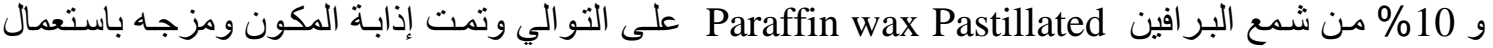

مذيب xylolzul analyse وبدرجة حرارة مزج

غسل الكاؤولين للتخلص من الأمسلاح الذائبة و الثـوائب العضوية العالقة، بعد ذللك تمت عمليـة التجفيف

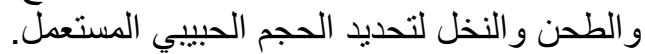

أضيف نسب 5\% و 10\% من كل من الخلطات المحضرة من الهرم الثناثي للتركيب اللائني إلى مسحوق

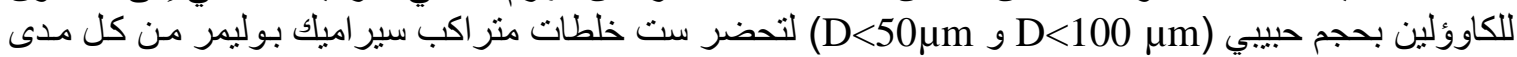

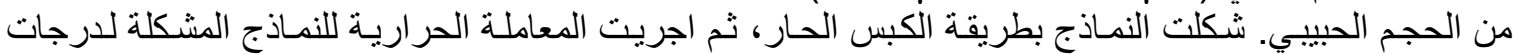

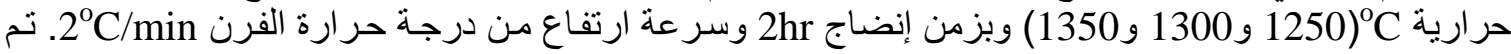

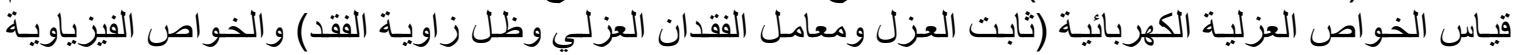
(التقلص الحبيي) لهذه النماذج المحضرة.

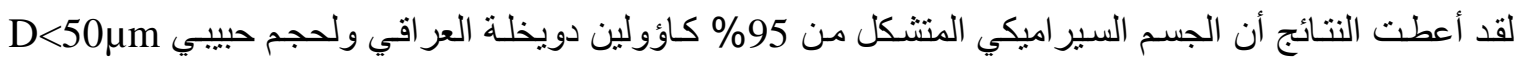

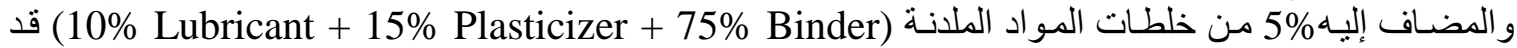
أعطت أفضل نتائج عند درجة حرارة حرق 1300م وكلما زادت نسب الإضافة [لحدود 10\%

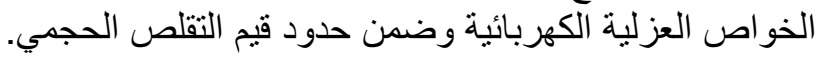

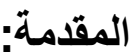

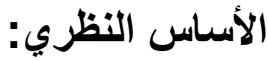

يمثنل الثـكل (1) علاقة مكونـات المسادة

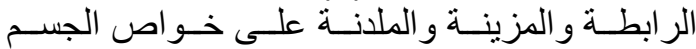
المشكل إذ يوضح الجذول (1) خصائص كل من هذه

المكونات:

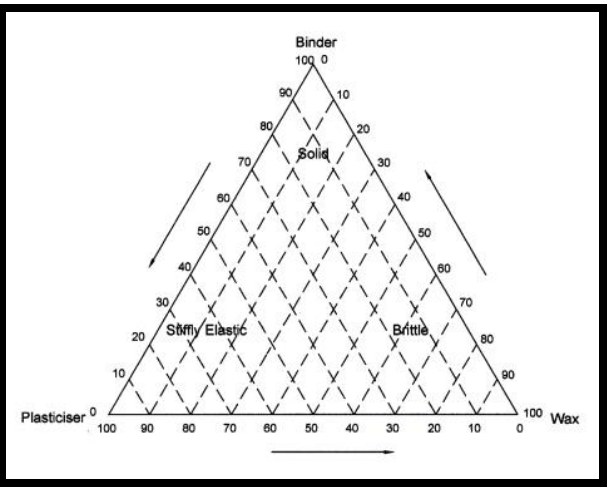

شكل (1) يوضح الهرم الثلاثي للمكونات (الملانة،

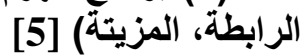

الجدول (1) يوضح خصائص المكونات الملانة [5]

\begin{tabular}{|c|c|c|c|c|}
\hline تأثير ها (عملها) & الخاصية & المركب & نوع المادة & \\
\hline وذات تأثرية و جيئة & قوة ميكانيكية & بولي فيانيل & المادة الر ابطة & 1 \\
\hline
\end{tabular}

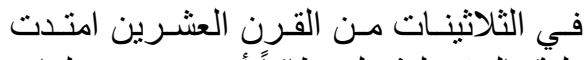

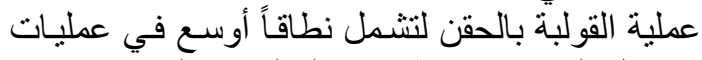

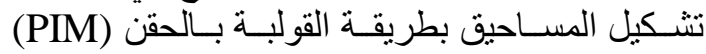
powder Injection molding

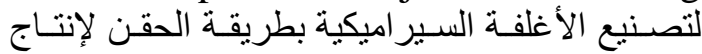

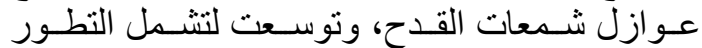

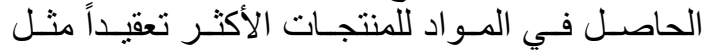

مقومات الأسنان و انتهاء بالتغليف الإليكتروني [1].

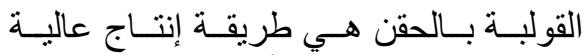
الإنتاجيـة واطئة الكلفة، لتشكيل أجز اء ذات ذات تكر اريـة

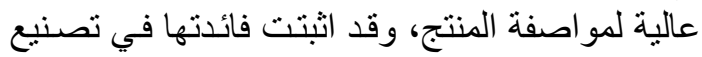

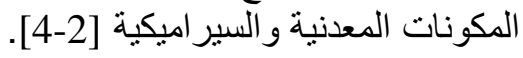

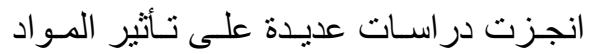

الر ابطة والمزينة و الملدنة على التشكيل بـالحقن - 8)

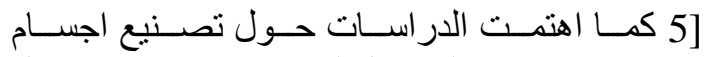

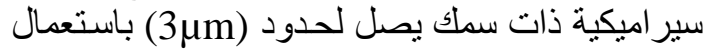



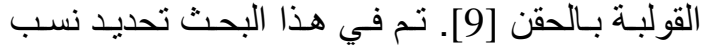

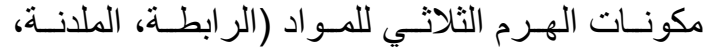

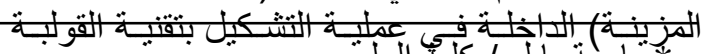

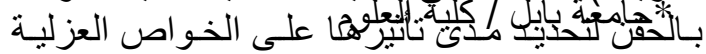

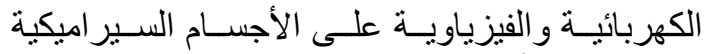
و المشكلة من أطيان محلية. 


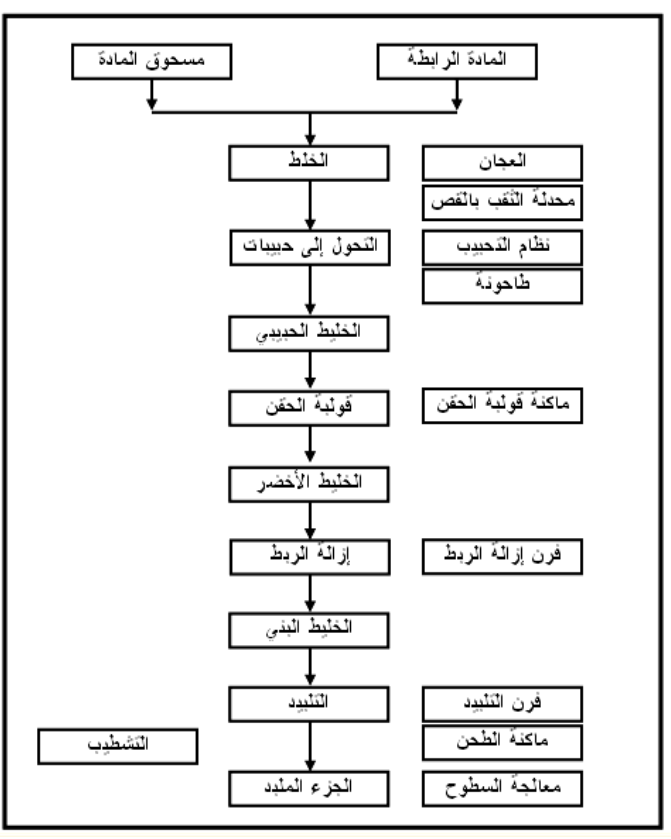

شكل (2): يوضح مراحل تحضير الجسم السيراميكي

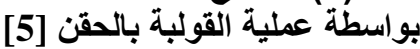

ان الظاهرة الناتجة من الحرق تسمى بالتلبيد

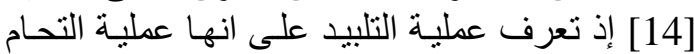

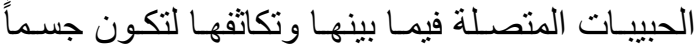

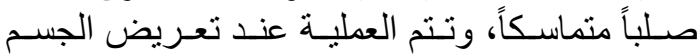

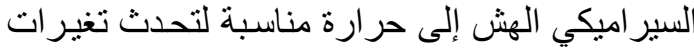

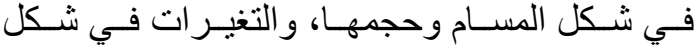

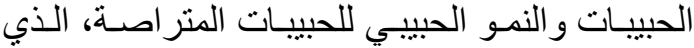

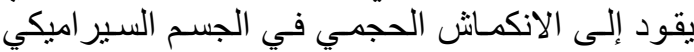

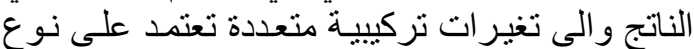

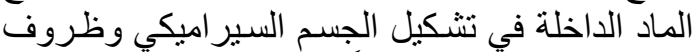

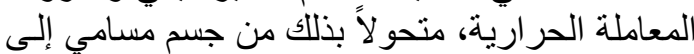

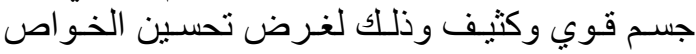

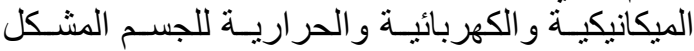

ان مفهوم الخو اص الفيزيائية تعني التقلص

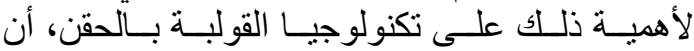

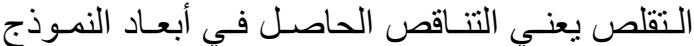



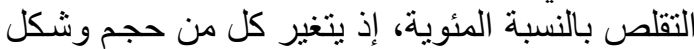

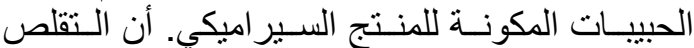
نوعان: التقلص الخطي (linear Shrinkage) وهو النو

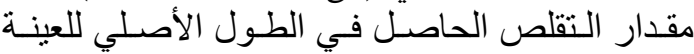

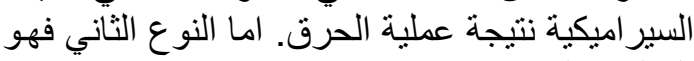

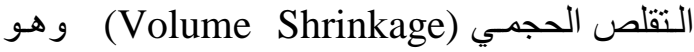

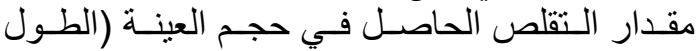
و السمك) نتيجة عملية الحرق [20].

هنالك عدة عوامل عدة تؤثر في مقدار التقلص منها

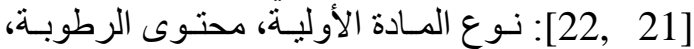

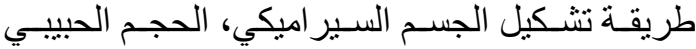

\begin{tabular}{|c|c|c|c|c|}
\hline $\begin{array}{c}\text { للتشققات } \\
\text { (Good } \\
\text { retention of } \\
\text { shape, low } \\
\text { susceptibility } \\
\text { to cracking) }\end{array}$ & $\begin{array}{c}\text { عالية } \\
\text { (High } \\
\text { mechanical } \\
\text { strength) }\end{array}$ & $\begin{array}{c}\text { بيوتير ال } \\
\text { (Polyvinyl } \\
\text { butyral) }\end{array}$ & (Binder) & \\
\hline $\begin{array}{c}\text { ونشطلة تثذيب } \\
\text { وتشطيب } \\
\text { (Easy for } \\
\text { trimming and } \\
\text { finishing) }\end{array}$ & مطاط صلب & $\begin{array}{c}\text { حامض فثالك } \\
\text { أسنر } \\
\text { (Phathalic } \\
\text { acid ester) }\end{array}$ & المادة الملدنة & 2 \\
\hline $\begin{array}{l}\text { اقتصادي في الحقن } \\
\text { (Economic to } \\
\text { injection) }\end{array}$ & $\begin{array}{c}\text { هrittle) } \\
\text { (B) }\end{array}$ & $\begin{array}{l}\text { وصع طبيعي } \\
\text { وصيعي } \\
\text { (Natural } \\
\text { and } \\
\text { Synthetic } \\
\text { wax) }\end{array}$ & المادة المزيتة & 3 \\
\hline
\end{tabular}

يمكن استـعمال معظم المـو اد القابلـة للتلبيـــ

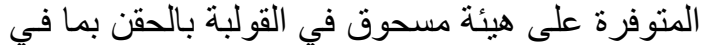

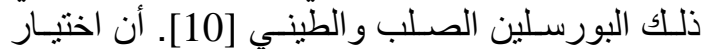

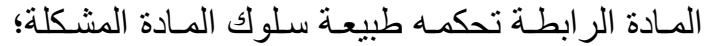

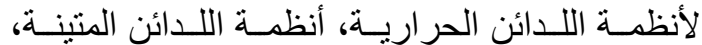

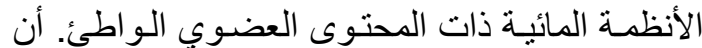
الأنظمة اللائنية الحراريـة هي التي يغلتب التئية استعمالها

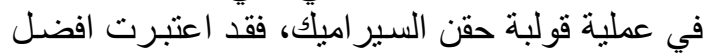

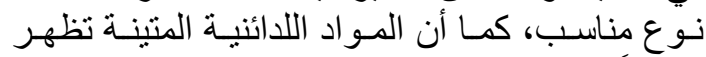

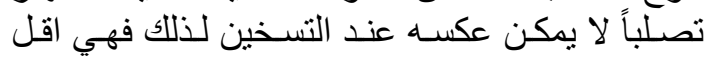

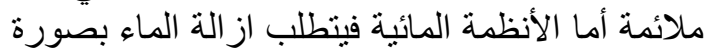
جزئية اثناء الحقن بو اسطة جدار قالب مسامي [5].

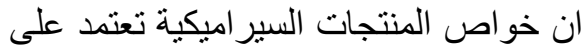

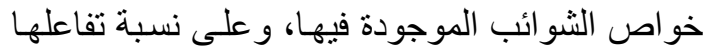

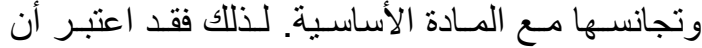

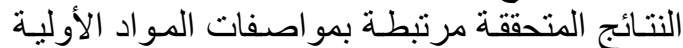

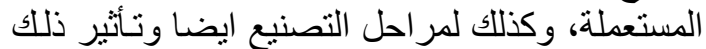

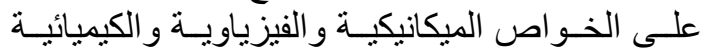


مر احل تحضير الجسم السير اميكي بالقولبة بالحقن.

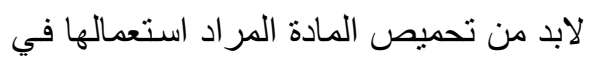

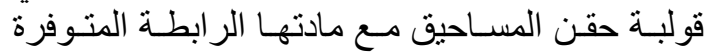

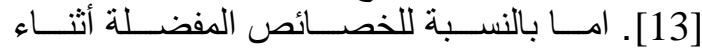
التحميص والتلبيد هو الوصول إلى خليط ذي انتفاخ التئ

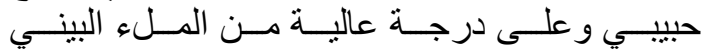
لتقليـل الإنكمـاش ومقاومــة التهـة (Interstitial Filling) التصدع أثناء التلبيد [5]. 
تتأثر قيمـة ثابت العزل وقيم معامل الفقدان

العزلي بالتغير ات التركيبية، وطيت وطريقة التصنيع، الكثافة المسامية، تردد الفولتية المسلط [27-29].

كما أن فقدان الطاقة في الأجسام السير اميكية

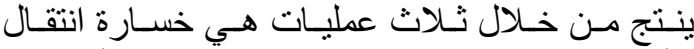

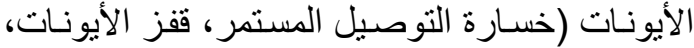

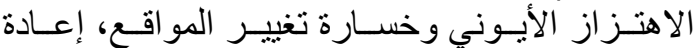

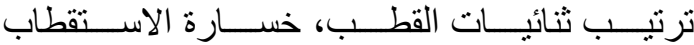
الإليكتروني). إذ تعرف العمليتان الأولى و الثانية على الإلى أنها التسرب الحاصل في التيار.

$$
\text { الجزء العملي : ابل }
$$

تـم اختيـار مـو اد محليـة عر اقيـة ( كـاؤولين


التحليل الكيمياوي لهذه المادة،

جدول (2) يوضح التحليل الكيميائي كاؤولين

دويخلة

\begin{tabular}{|c|c|c|c|}
\hline الأوكسيد & $\begin{array}{c}\text { النسبة المئوية } \\
\text { (\% }\end{array}$ & نوع الأوكسيد & 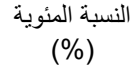 \\
\hline $\mathrm{SiO}_{2}$ & 52.35 & $\mathrm{MgO}$ & 1.11 \\
\hline $\mathrm{Fe}_{2} \mathrm{O}_{3}$ & 1.31 & $\mathrm{SO}_{3}$ & 0.45 \\
\hline $\mathrm{Al}_{2} \mathrm{O}_{3}$ & 34.02 & $\mathrm{Na}_{2} \mathrm{O}$ & -- \\
\hline $\mathrm{TiO}_{2}$ & 0.12 & $\mathrm{~K}_{2} \mathrm{O}$ & -- \\
\hline $\mathrm{CaO}$ & 1.2 & L.O.I & 12.54 \\
\hline
\end{tabular}

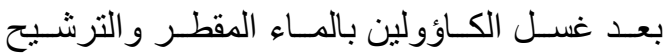

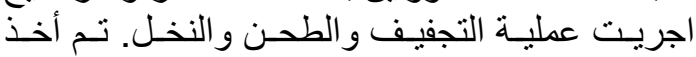

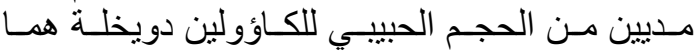

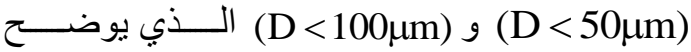

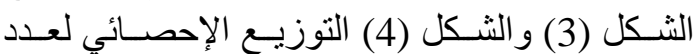

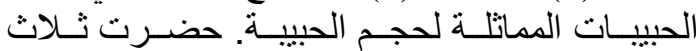

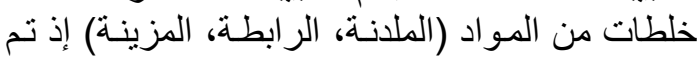

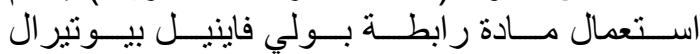
Polyveniyl Butyral ومادة ملدنة حامض فيثالك بئك ايستر Di- n-butyl phathalate ومادة مادة مزيتة

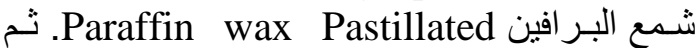

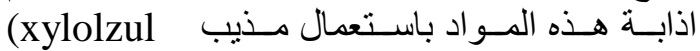
(analyse) المستعملة لتحضير المجاميع الثثلاثة:

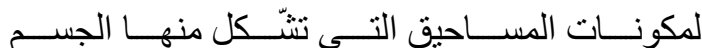
السير اميكي، مدى فعالية تحرير غاليكاز التهات اثناء المعاملة

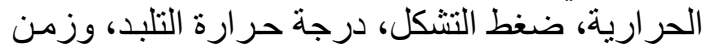
الحرق ومعدل ارتفاع درجة حر ارة الفرن.

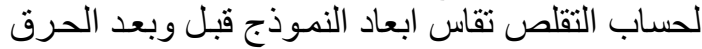

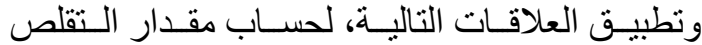

$$
\text { بنو عيه [24, 23, 23, }
$$

linear shrinkage (L.Sh.) $=\frac{\mathrm{L}_{0}-\mathrm{L}}{\mathrm{L}_{0}} \times 100 \% \cdots(1)$

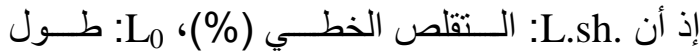

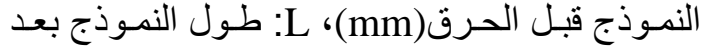

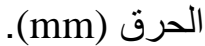

Volume Shrinkage (V.Sh.)

$$
\begin{aligned}
& =\frac{\mathrm{V}_{0}-\mathrm{V}}{\mathrm{V}_{0}} \times 100 \% \cdots(2) \\
& \text { V. sh . }=\left(1-\frac{D^{2} \ell}{D_{0}^{2} \ell}\right) \times 100 \%
\end{aligned}
$$

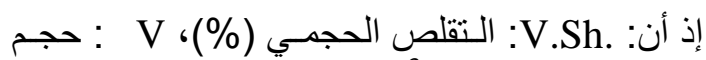

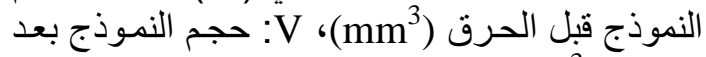

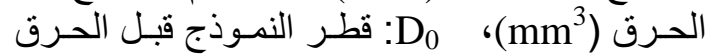

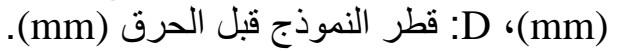

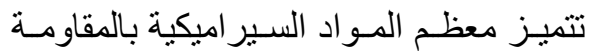

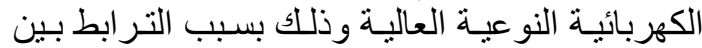



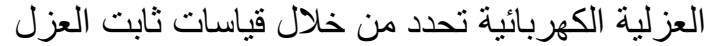

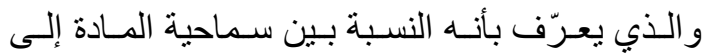
سماحية الفراغروفي ويحسب من العلاقة [26, 25]: $\epsilon^{\prime}=\left(\frac{1}{\epsilon_{0}}\right)\left(\frac{d}{A} C\right) \quad \cdots$

إذ أن d: سمك النموذج (mm)، Am) المساحة الفعالة

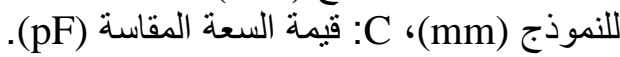

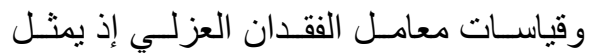

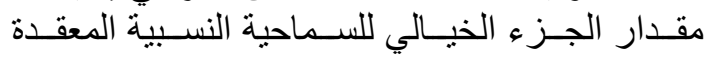
ويحسب من العلاقة التالية [30, 28]:

$\mathrm{k}^{\prime \prime}=\left(\frac{1}{\mathrm{R}_{\mathrm{p}} \mathrm{w} \in_{0}} \frac{\mathrm{d}}{\mathrm{A}}\right)$

: هي القيمة المقاسة لمقاومة المادة Re

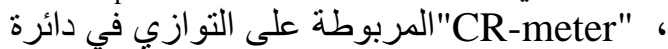

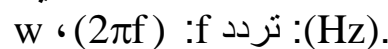




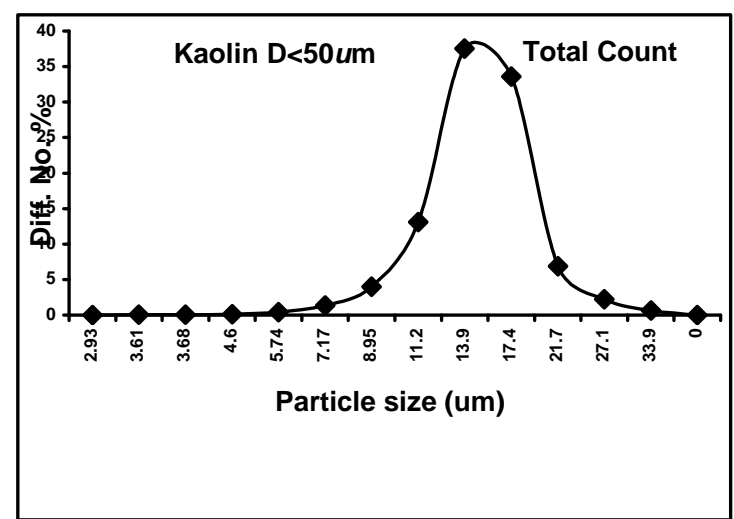

شكل (3) التوزيع الحبيبي لحبيبات الكاؤولين

$(\mathrm{D}<100 \mu \mathrm{m})$

جدول (4) يوضح النسب والمكونات الداخلة في

تكوين المخلطات من كاؤولين دويخلة

\begin{tabular}{|c|c|c|c|}
\hline \multirow{2}{*}{ الخلطة } & \multicolumn{2}{|c|}{ المو اد الملانة } & كاؤولين دويخلة \\
\hline & جدم الجموعة & 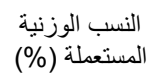 & الوزنيّة(\%) \\
\hline $\mathrm{C}_{1}$ & $\mathrm{R}_{1}$ & 10 & 90 \\
\hline $\mathrm{C}_{2}$ & $\mathrm{R}_{2}$ & 10 & 90 \\
\hline $\mathrm{C}_{3}$ & $\mathrm{R}_{3}$ & 10 & 90 \\
\hline $\mathrm{C}_{4}$ & $\mathrm{R}_{1}$ & 5 & 95 \\
\hline $\mathrm{C}_{5}$ & $\mathrm{R}_{2}$ & 5 & 95 \\
\hline $\mathrm{C}_{6}$ & $\mathrm{R}_{3}$ & 5 & 95 \\
\hline
\end{tabular}

جدول (5) يوضح النسب والمكونات الداخلة في D > تكوين المخلطات من كاؤولين دويخلة

\begin{tabular}{|c|c|c|c|}
\hline \multirow{2}{*}{ الخلطة } & \multicolumn{2}{|c|}{ المو اد الملدنة } & كاؤولين دويخلة \\
\hline & جدم المجمو عة (5) & المستعملة (\%) & $\begin{array}{c}\text { النسب الوزنية } \\
\text { (\%) }\end{array}$ \\
\hline $\mathrm{D}_{1}$ & $\mathrm{R}_{1}$ & 10 & 90 \\
\hline $\mathrm{D}_{2}$ & $\mathrm{R}_{2}$ & 10 & 90 \\
\hline $\mathrm{D}_{3}$ & $\mathrm{R}_{3}$ & 10 & 90 \\
\hline $\mathrm{D}_{4}$ & $\mathrm{R}_{1}$ & 5 & 95 \\
\hline $\mathrm{D}_{5}$ & $\mathrm{R}_{2}$ & 5 & 95 \\
\hline $\mathrm{D}_{6}$ & $\mathrm{R}_{3}$ & 5 & 95 \\
\hline
\end{tabular}

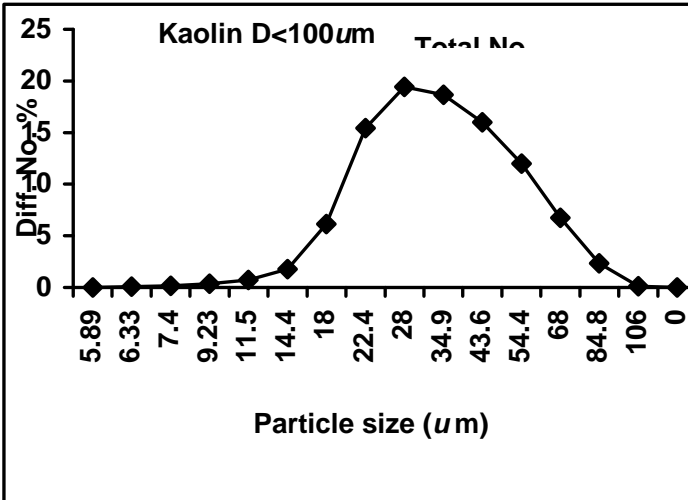

شكل ( 4) التوزيع الحبيبي لحبيبات الكاؤولين

$(D<50 \mu m)$

جدول (3) يوضح نسب مكونات الخلطات (الملانة، الر ابطة، المزيتة)

\begin{tabular}{|c|c|c|c|c|}
\hline \multirow{2}{*}{ المجموعة رمز } & $\begin{array}{c}\text { المادة الرابطة } \\
\text { (B) } \\
\text { (Binder) }\end{array}$ & $\begin{array}{c}\text { الملدادة } \\
\text { (P) } \\
\text { (Pasticiz } \\
\text { er) } \\
\end{array}$ & $\begin{array}{c}\text { المادة المزيتة } \\
\text { (L) } \\
\text { (Lubricant) }\end{array}$ & المادة المزيتة \\
\hline & $\begin{array}{c}\text { Polyviny } \\
\text { butyral }\end{array}$ & $\begin{array}{l}\text { Phathali } \\
\mathrm{c}^{* 2} \text { acid } \\
\text { ester }\end{array}$ & $\begin{array}{l}\text { Paraffin }{ }^{* 1} \\
\text { Wax } \\
\text { Pastillated }\end{array}$ & $\begin{array}{c}\text { Xylolzul }^{* 3} \\
\text { Analyse }\end{array}$ \\
\hline $\begin{array}{c}\text { الوزنية } \\
\text { النسبة } \\
\end{array}$ & $\begin{array}{c}\text { النسبة الوزنية) } \\
\text { (\%) }\end{array}$ & $\begin{array}{c}\text { الوزنية } \\
\text { (\%) } \\
\end{array}$ & $\begin{array}{c}\text { النسبة الوزنية } \\
\text { (\%) }\end{array}$ & $(\mathrm{ml})$ \\
\hline $\mathrm{R}_{1}$ & 70 & 20 & 10 & 100 \\
\hline $\mathrm{R}_{2}$ & 75 & 15 & 10 & 100 \\
\hline $\mathrm{R}_{3}$ & 80 & 10 & 10 & 100 \\
\hline \multicolumn{5}{|c|}{$\begin{array}{l}* 1 \text { : Parnffin wax pastilled BDH Laboratory supplies. } \\
* 2 \text { : Industrical product. } \\
* 3 \text { : XylolZur analyse (isomeren gemisch } \mathrm{C}_{8} \mathrm{H}_{10} \text { ). }\end{array}$} \\
\hline
\end{tabular}

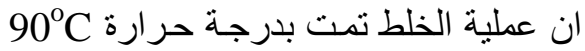

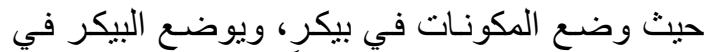

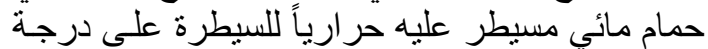

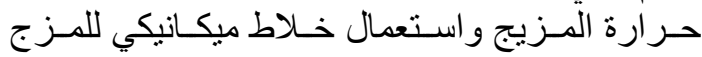
بريشة زجاجية.

اخذت نسبة 5\% و و10\% من مجاميع المواد

الملدنة $)$ و أضيف إلى كاؤولين دويخلة

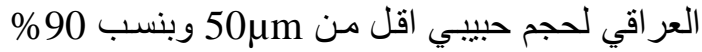
و95\% من حجم المكون المخلوط، وكمـا يوضـحها

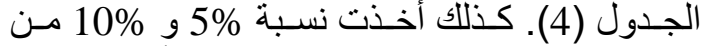
مجاميع المواد الملدنة $)\left(\mathrm{N}_{3}, \mathrm{R}_{2}, \mathrm{R}_{1}\right.$ و أضيفت إلى

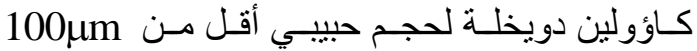
وبنسب 90\% و 95\%، كما يوضحها الجدول (5). 
للتقلص أقل من استعمال مسحوق كاؤولين معرف بالثكل (3) ولمدى حجم حبييـي (D رغم تو افق سلوكه مع زيادة المادة الر ابطة.

يوضح الثكل (8,7) سلوك خاصيتي ثابت

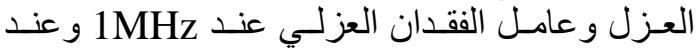

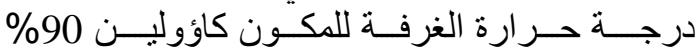
905 9 و مضـاف إليـه الدكونـات البوليمريـة $(70 \% \mathrm{~B}+20 \% \mathrm{P}+10 \% \mathrm{~L})$ $(75 \% \mathrm{~B}+15 \% \mathrm{P}+10 \% \mathrm{~L})$, لكـل مكسـون علــ (80\% B+10\%

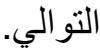

إذا أخذنا حجم الحبييات منساو فأن المساحة

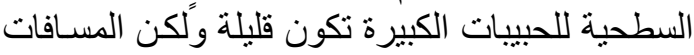

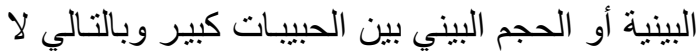

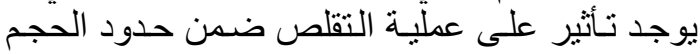
الفر اغي بين الحبييات و إنما ينحكم بالتقلص هو فاعلية

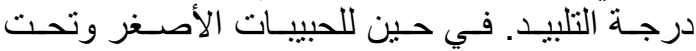
افتر اض تساوي الحجم فأن الدساحة السطحية تكون

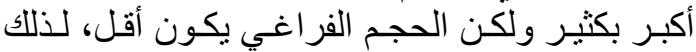


الفر اغي بين الحبيبات ولكن لمدى أقل من الأكبر ،

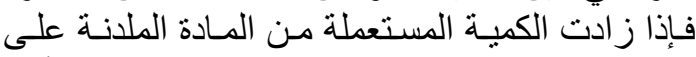

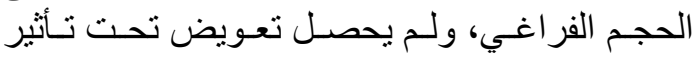

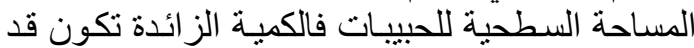

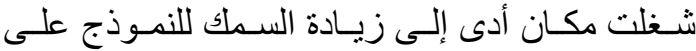



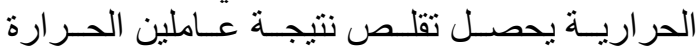
(التلبيد) وخروج المادة الملدنة.

ولكن عند استعمال مسحوق ذو حبيبي و اطئ

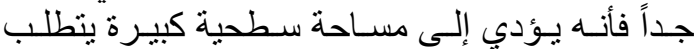

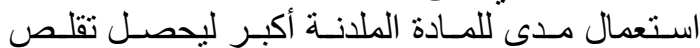
مؤثر. أن هذا التصور بلى مثنانياً في النتائج نتيجة

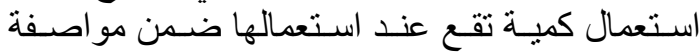

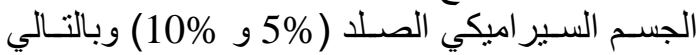

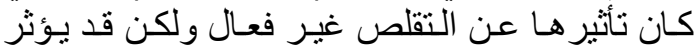

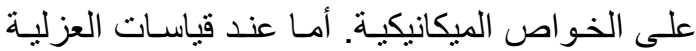

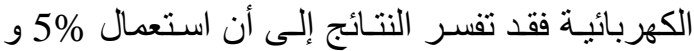
10\% من المو اد الملدنة ساعد على انزلاق الكي الحيبيات فيما بينها وحقق تثكيل قوى أدى إلى عمليات تلبيد قد اليد



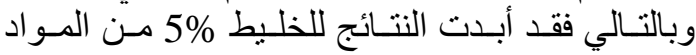

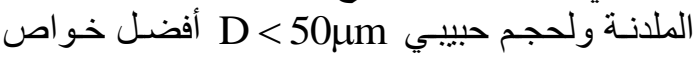

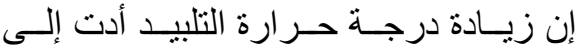

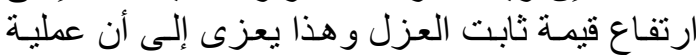

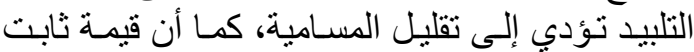

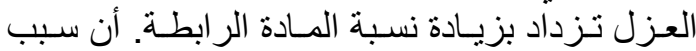

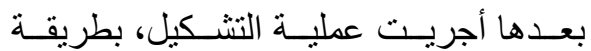

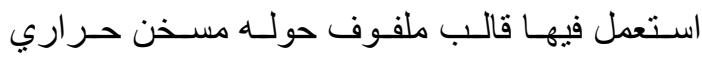

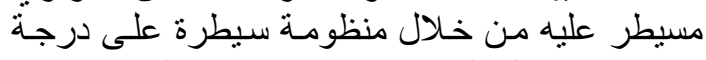

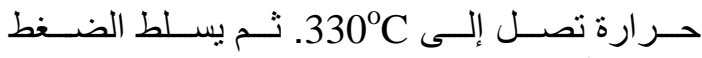



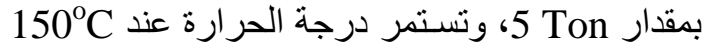

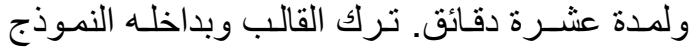
لييرد للارجة حرارة تتراوح بين (30 - 20) دقيقة بعد ذلك تخرجة العرة العينة

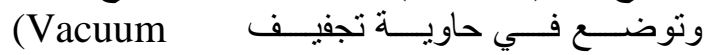

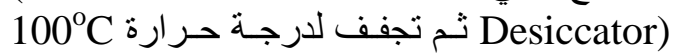

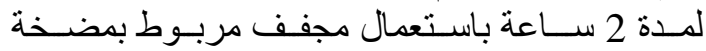

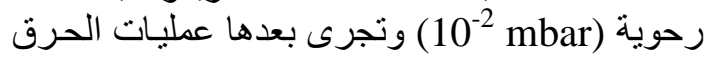


وزمن انضاج 2hr ومعدل ارتفاع في درجة الحرارة $.2^{\circ} \mathrm{C} / \mathrm{min}$

\section{النتائج والمناقشة:}

تم قياس التقلص الحجمي وذلك بقياس ابعاد

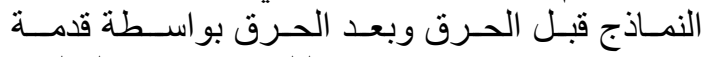
وبعد ذلك تم حسـاب التقلص (vernier caliper)



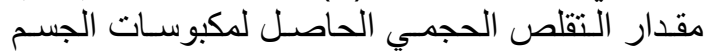

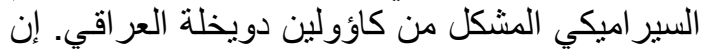

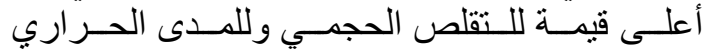

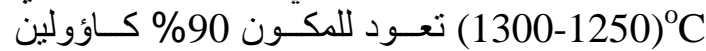

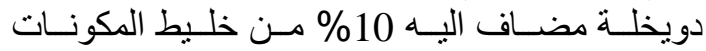



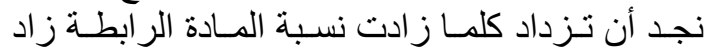

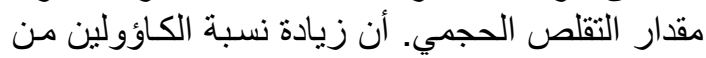
90 \% إلى 95\% ولنفس المدى الحبيبي قد ادى إلى إلى إنى

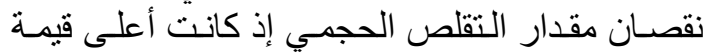

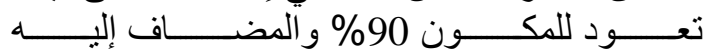
80\%B+10\%P+10\%L .$(1250-1350)^{\circ} \mathrm{C}$

كمـا أن مقدار التقلص الحجمي يتغيـر تبعـاً

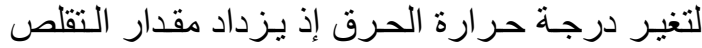

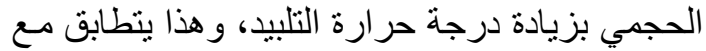

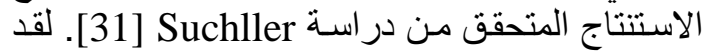

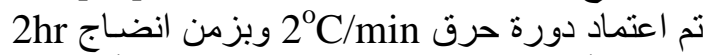

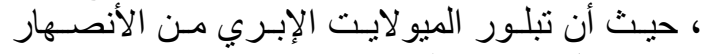

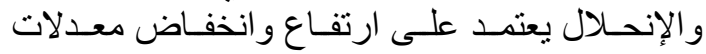

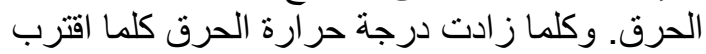

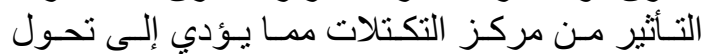

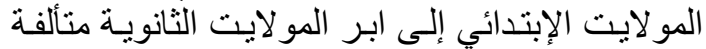

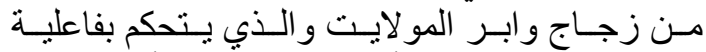
التقلص الحجمي. من الأشكال البيانية نجد أن استعمال

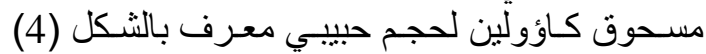

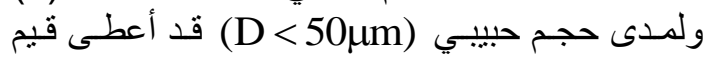


من حيث التشكيل ومضافات الهرم الثلاثي للمكونات

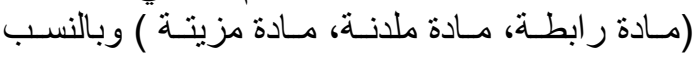
المحددة 90\% و 95\% من مكون الكاؤولين ممزوج

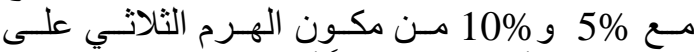

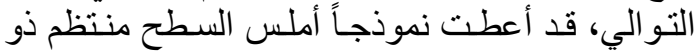

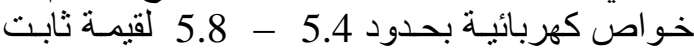
العزل و (0.04 - 0.05) لعامل الفقدان للنموذجين

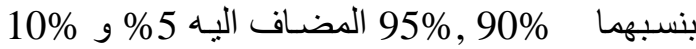

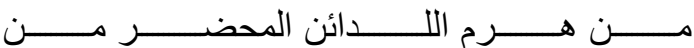
10\%L+15\% P+75\%B]

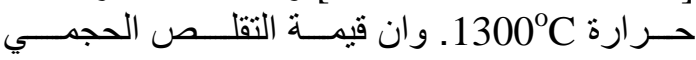
المقاس عند هذه الظـروف كانت لحدود $.15) \%$

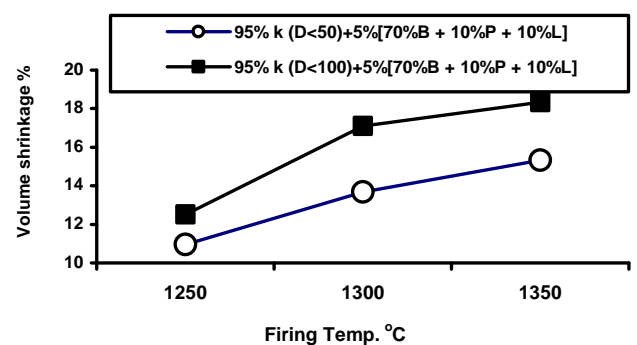

( ( )

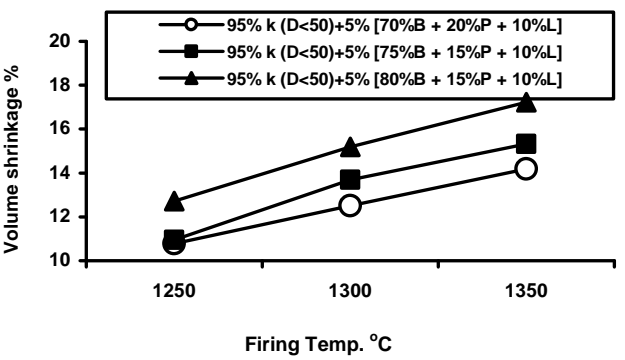

(ج)

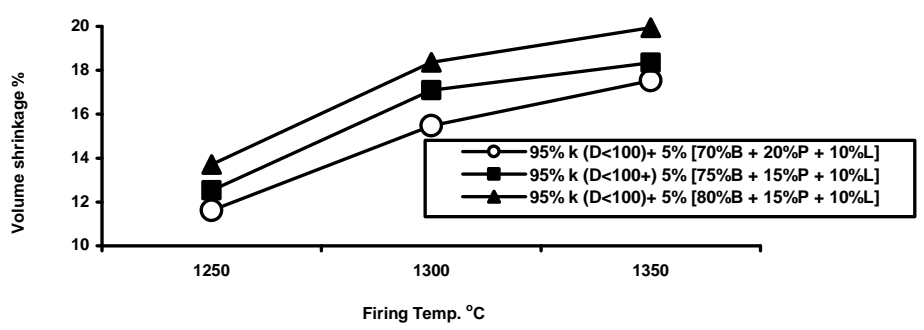

$\rightarrow$

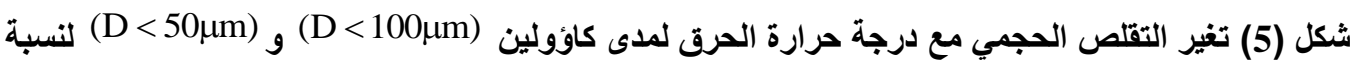

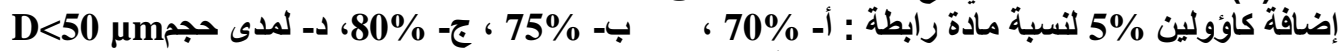

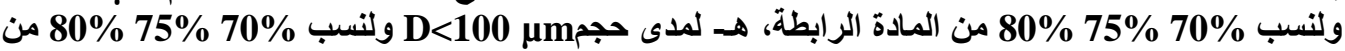



تغيـر ثابـت العزل مــ درجـة الحـر ارة يسبب تحفـز

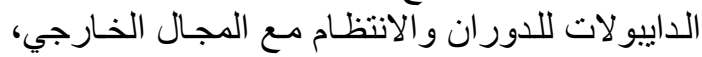

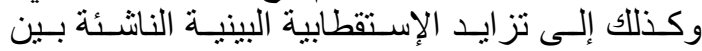

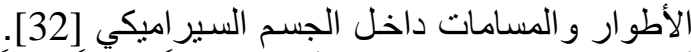





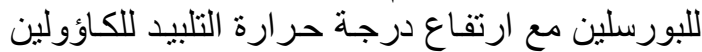

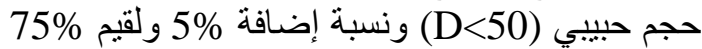
من المادة الر ابطة.

الاستنتاج:

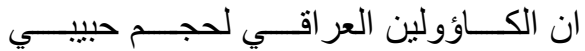
D D $250 \mu \mathrm{m}$

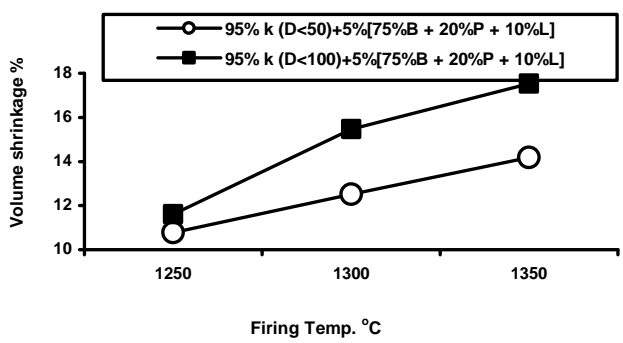

$(ب)$

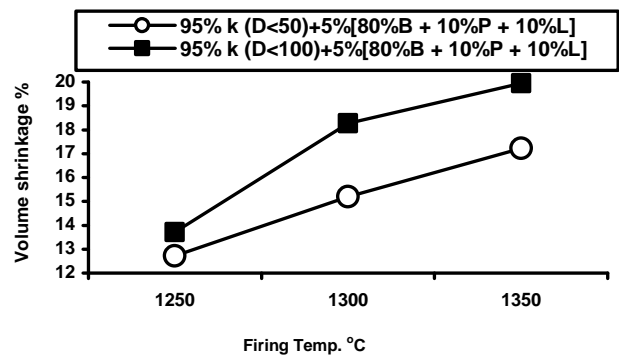

$(د)$ 




(أ)

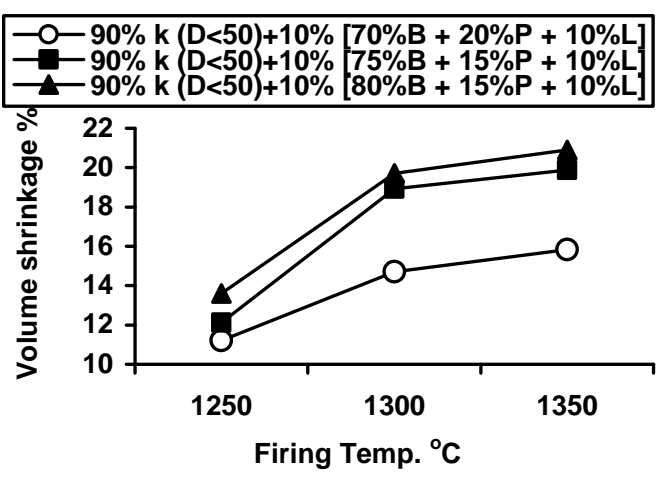

(ج)

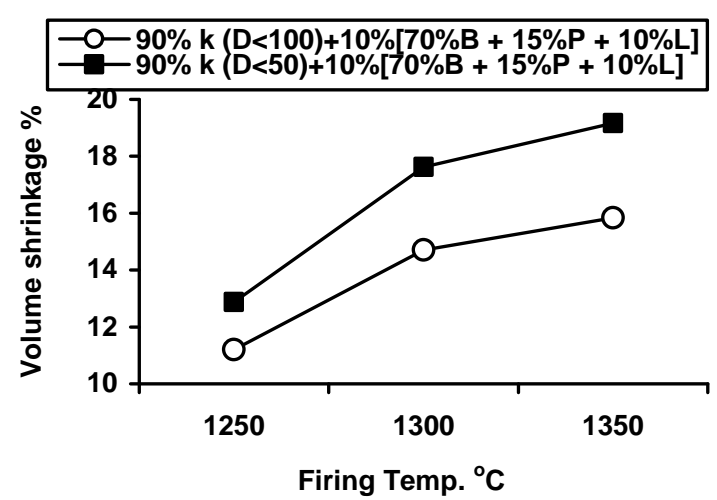

$(ب)$

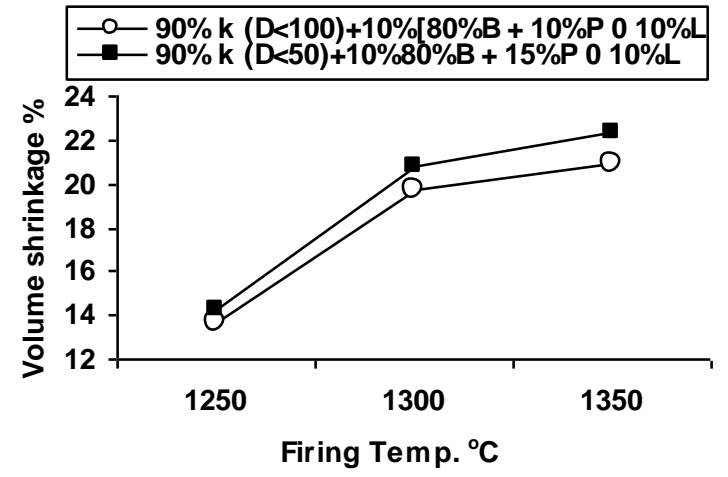

$(د)$

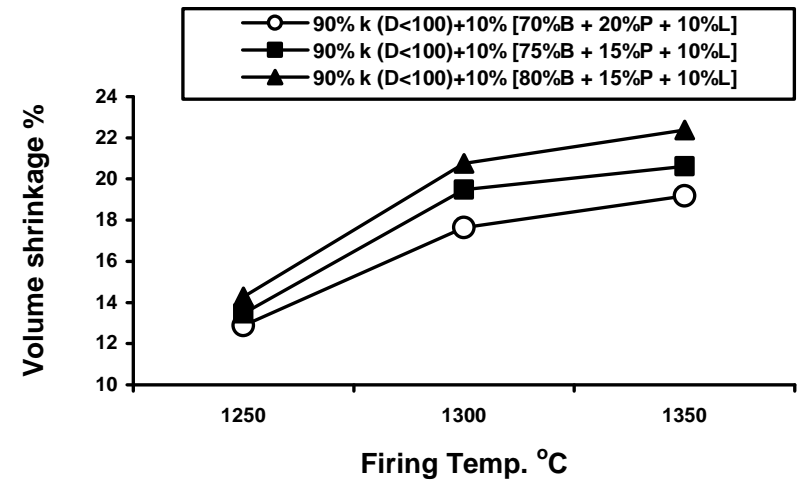

$(\rightarrow)$

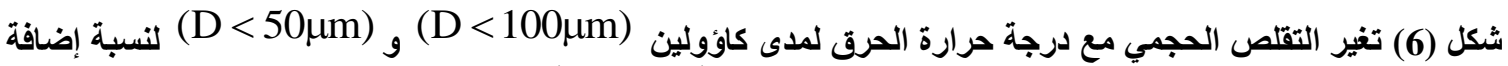
كاؤولين $10 \%$

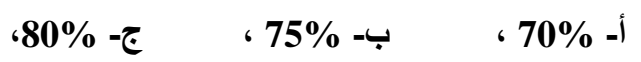

دـ لمدى حجم D>50 ولنسب 70\% 75\% 80\% من المادة الرابطة،

هـ لمدى حجم D<100 ولنسب $70 \%$ 75\% $70 \%$ من المادة الرابطة 


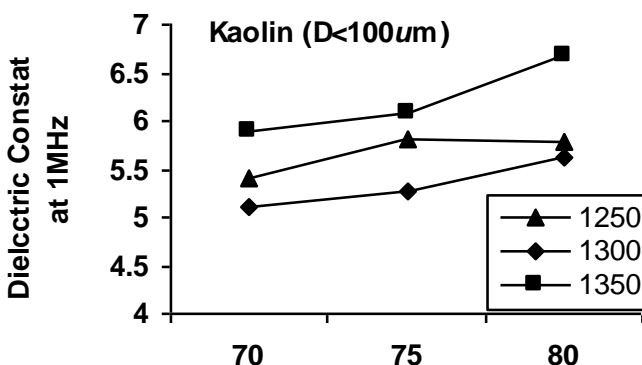

$5 \%[x$ Binder concentration]

(ا)

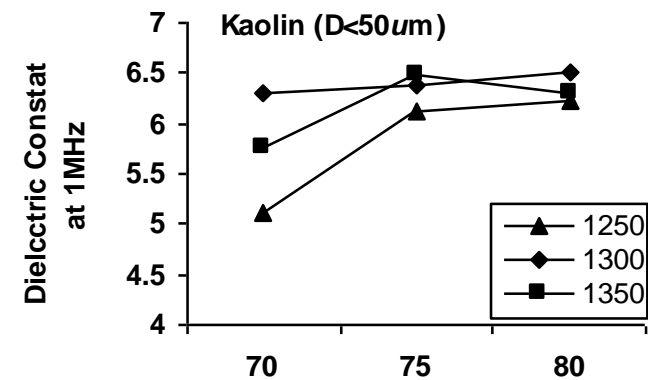

$5 \%[x$ Binder concentration]

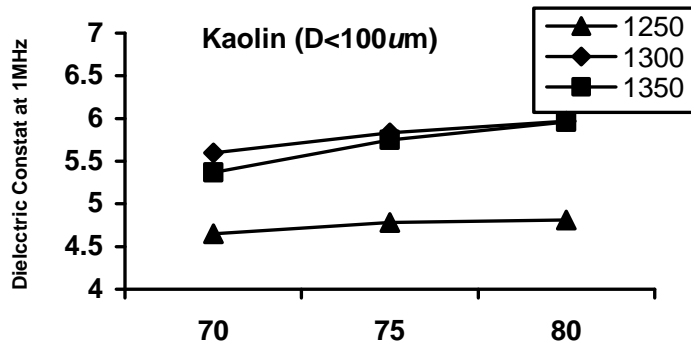

$10 \%[x$ Binder concentration $]$

(ب)

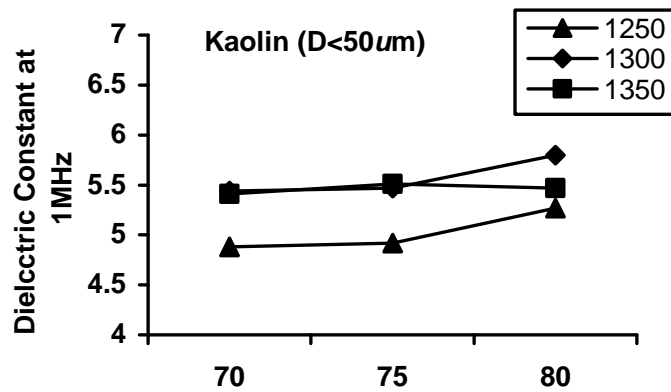

$10 \%[x$ Binder concentration]

(ج)

(د)

شكل (7) تغير قيم ثابت العزل عند 1MHz لارجات حرارية مختلفة مع تركيز المادة الرابطة لنسبة إضافة 5\% و 10\%

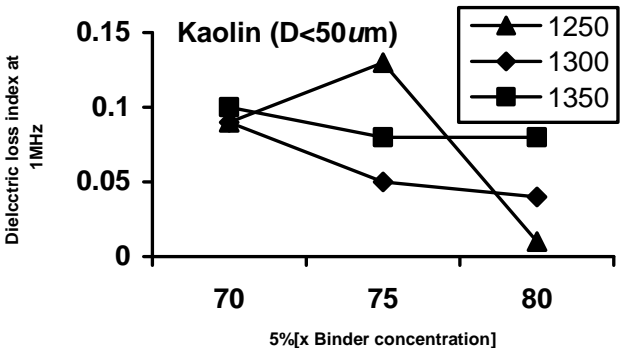

(أ)

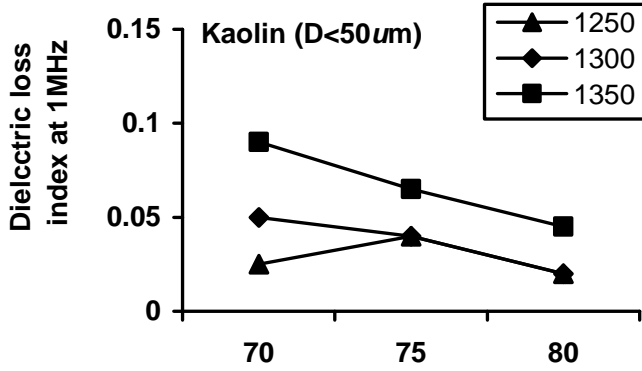

$5 \%[x$ Binder concentration $]$

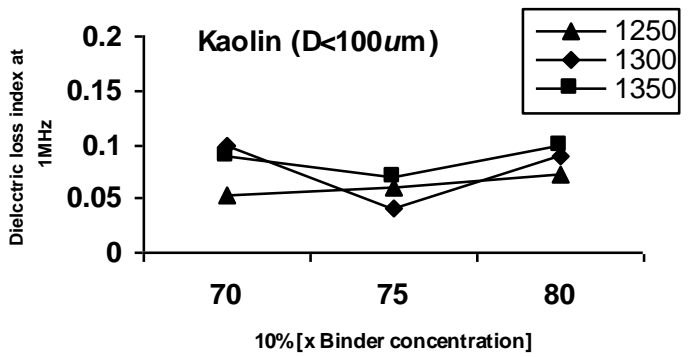

(ب)

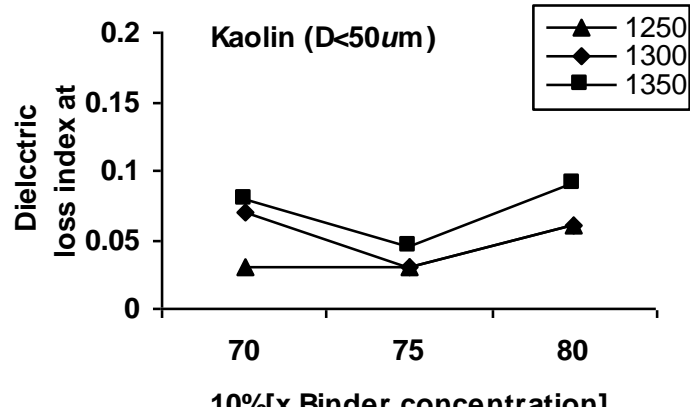

$10 \%[x$ Binder concentration]

\section{(ج)}

شكل (8) تغير قيم ثابت العزل عند 1MHz لارجات حرارية مختلفة مع تركيز المادة الرابطة لنسبة إضافة 5\% و 10\% 
ceramic", (Sh. Somiya. ed). Academic press, Inc, San Diego California:75-81.

15. Coble, R. L. (1961) "Sintering Crystalline solids", Inter- mediate and final State diffusion models in powder compacts" J. of Applied. Phys., 32(5):787-792.

16. Coble, R. L. (1961) "Sintering Crystal line solids", experimental test of diffusion models in powder compacts", J. of Applied physics, 32(5):793-799.

17. Lang, F.F and Kellet, B. (1985) "Influence of particle arragesment on sintering", Science of ceramic. (L.L Hench and D.R. Ulriched). Awileyinters science publication, john Wiley \& sons, New York,.

18. Cohen M. \& Norton F.H (1964)" [physical ceramic for engineering, by Addison- wesely publishing company, 99-101.

19. Kingery, W.d., and Morris, B.(1955)"The study of sintering isolids by viscous flow, evaporation- conden sati and self, diffusion", $\mathrm{J}$ of -Applied phsics, 26:1250-1212.

20. Andrews, Al. (1957) "Ceramic Test and cakulations" $9^{\text {th }}$, edition, by john Wiley \& Sons, inc.:17-30.

21. Huggins, J. (1996) " Minaly Amied At the porcelain Industry". Inter ceramic, 45:115-117.

22. K.ngery ,W.D. (1967) "Introduction to ceramic", co. D Bowen, H.K. and D.R, $2^{\text {nd }}$ edition, john wiley, and Sons, New York,:1032.

23. ASTM, (1988):326-82.

24. Griffilths, R. \& Raeford, C. (1965)" Calculation in ceramic", Meclaren \& Sons, LTD.

25. Zbigniew, D. jastrzebski, (1986)"The nature and Properties of Engineering Materials", Vol. 2, $2^{\text {nd }}$ edition, John Wiley and Sons Ltd., Malta,

26. Tareev, B., (1979) "Physics of Dielectric materials", Mir Publishers, Moscow, pp. 13 - 16, 44 - 48.

27. ASTM Designation D150-68: Standard method of Test for A.C. Loss Characteristics and Dielectric Constant
1. Germany, R. M, (1989) "

Arationalization of the powder injection moulding pricess for stainless steels based on component features", proc Int, Conf, on powder Metallurgy \& particulate Material, Las Vegas, Nv.

2. Tegman,R. (1985) "Hot isostice pressing of silicon Nitride", Inter ceramic, 1:23-27.

3. Alcock , J. and Stephenson,D. (2003) "The powder Injection moulding process", Materials world:629-630.

4. Rado, P. (1964) " An Introduction to the technology of pottery", $2^{\text {nd }}$ Edition by pergamon press.

5. Saure ,F. (1984)" Injection moulding" Inter ceramic, 33:1-2.

6. Faneeli, A.J., Silvers, R.D, et. al, (1984) " Powder injection moulding of ceramics parts" J. of Arner. Ceram. Soci, 72(10)87-92.

7. Peter R. (1995)." An approach to cost Efficient preparation of ceramic injection - moulding compounds", Inter ceramic, 44( 4):248-250.

8. Clifford P. (2001). "Powder injection moulding", ceramic Technology for Broad Based manufacturing NIST, ATP, ( 93):106-113.

9. Volker P,and Gietzet,T(2003) "Micropowder- injection moulding of etals and ceramic", Sadhand, 28 (1\&2):299-306.

10. Practice \& Management (1995) " Injection moulding of porcelain", Inter cerac, 44(2):86-87.

11. Hech L.I. \& Could R.W., (1971)"Characterization of ceramic", Marcel Dekker Inc, New York,

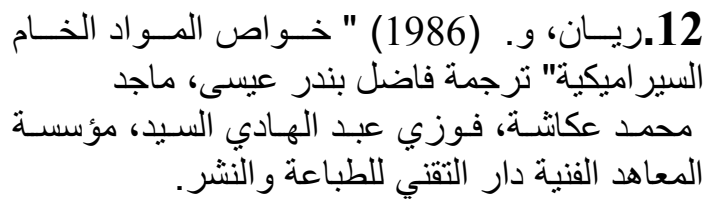

13. Gerguis A. and Husein,A. (1983)"

Chemistry and technology of polymers"462-466.

14. Fukuura I.(2001) " Production process for ceramic', Advanced technical 
30. ASTM Designation D1711-70: standard Definitions of Terms Relating to Electrical Insulation,:728.

31. Schuller, K. H. (1964) Trans Brit. Cerman. Soc., Vol. 63:

32. Kinser, D. L., (1971) "Electrical Conduction in Glass and Glass Ceramics. Physics of Electronic Ceramics, Part A, (L. L. hench and D. B. Dove ed.), Marcel Dekker, Inc., New York.
(Permittivity of Solids Electrical Insulating materials.

28. Harrop, P. J., (1972) "Dielectrics Butter Worth and Co., Publishers, Ltd. London, Butter Worths.

29. Clarck F.M, (1962)" Insulating material for Design and Engineering practices". By john wiley \& Sons, Inc, pp [34, 36, 39].

\title{
Effect of Adding (Binder-Plastisizer-Lubricat) on the Physical Properties of Kaolin
}

\author{
Fadial A. Rasin*
}

*College of Science $\backslash$ Babylon University

\begin{abstract}
:
The study concern with the preparation of three type of mixtures; which are prepared from different percentage of polyvenil Butyral, Di-n-butyl phathalate and paraffin wax pastillated. The solvent used is Xylolzul analyses.

After washing, Drying and milling the kaolin Dukhla, as a matrix in this study, and by using sieving Tech. The range of particle size used is less than $100 \mu \mathrm{m}$ and less than $50 \mu \mathrm{m}$ as a mesh batch. The added percentage from prepared mixture were 5\% and $10 \%$ to $95 \%$ and $90 \%$ of the matrix respectively. Then disk samples were prepared by using a compaction pressure with heating. After cooling and drying the samples were undergo heat treatment in the range of $(1250-1350){ }^{\circ} \mathrm{C}$.
\end{abstract}

The measurement of shrinkage and Dielectric properties shows that the satisfied data to be compatible with the industrial properties were for $5 \%(75 \% \mathrm{~B}+15 \% \mathrm{P}+10 \% \mathrm{~L})$ added to $5 \%$ to $95 \%$ of Kaolin (Particle size less than $50 \mu \mathrm{m}$ ); which was fired at $1300^{\circ} \mathrm{C}$. 